\title{
Chiral algebra of the Argyres-Douglas theory from M5 branes
}

\author{
Dan Xie, ${ }^{1,2}$ Wenbin Yan, ${ }^{1}$ and Shing-Tung Yau ${ }^{3,4,5}$ \\ ${ }^{1}$ Yau Mathematical Sciences Center, Tsinghua University, Beijing 100084, China \\ ${ }^{2}$ Department of Mathematics, Tsinghua University, Beijing 100084, China \\ ${ }^{3}$ Center of Mathematical Sciences and Applications, Harvard University, \\ Cambridge, Massachusetts MA02138, USA \\ ${ }^{4}$ Jefferson Physical Laboratory, Harvard University, Cambridge, Massachusetts MA02138, USA \\ ${ }^{5}$ Department of Mathematics, Harvard University, Cambridge, Massachusetts MA02138, USA
}

(Received 21 October 2016; accepted 9 February 2021; published 9 March 2021)

\begin{abstract}
We study chiral algebras associated with Argyres-Douglas theories engineered from M5 branes. For the theory engineered using $6 \mathrm{D}(2,0)$ type $J$ theory on a sphere with a single irregular singularity (without mass parameter), its chiral algebra is the minimal model of $\mathrm{W}$ algebra of $J$ type. For the theory engineered using an irregular singularity and a regular full singularity, its chiral algebra is the affine Kac-Moody algebra of $J$ type. We can obtain the Schur index of these theories by computing the vacua character of the corresponding chiral algebra.
\end{abstract}

DOI: 10.1103/PhysRevD.103.065003

\section{INTRODUCTION}

In the past few years, it has been found that certain observables of four-dimensional $\mathcal{N}=2$ superconformal field theories (SCFTs) can be identified with observables of two-dimensional conformal field theories. Those 4D/2D correspondences include the match between the 4D sphere partition function and the correlator of 2D Liouville theory [1], the match between the trace of 4D quantum monodromy and the character of certain module of $2 \mathrm{D}$ chiral algebra [2-5]. Recently, a remarkable map between 4D $\mathcal{N}=2$ SCFTs and 2D chiral algebras was constructed in [6]. In many cases, 2D chiral algebras constructed from 4D SCFTs are identified with known 2D models; see [5-13].

The purpose of this paper is to identify the chiral algebras corresponding to Argyres-Douglas (AD) theories [14] engineered from M5 branes [15-18]. The 2D chiral algebra is constructed from the 4D theory as follows. First, pick a two-dimensional slice in the four-dimensional space $\mathbb{R}^{2} \subset \mathbb{R}^{4}$ with complex coordinates $(z, \bar{z})$. Then choose a set of particular operators $\mathcal{O}(z, \bar{z})$ living on this plane $\mathbb{R}^{2}$ annihilated by a combination of Poincare and conformal supercharges $\mathfrak{Q}=Q+S$. At the origin, these operators are just Schur operators. The operator product expansion of these operators turns out to be meromorphic up to the $\mathfrak{Q}$-exact piece. Therefore, by passing through the

Published by the American Physical Society under the terms of the Creative Commons Attribution 4.0 International license. Further distribution of this work must maintain attribution to the author(s) and the published article's title, journal citation, and DOI. Funded by SCOAP ${ }^{3}$.
Q-cohomology, the operators $\mathcal{O}(z, \bar{z})$ form a meromorphic chiral algebra or vertex operator algebra (VOA). The basic 4D/2D mappings used in this paper are [6] ${ }^{1}$ as follows:

(i) The 2D central charge $c_{2 \mathrm{D}}$ and the level of affine Kac-Moody algebra $k_{2 \mathrm{D}}$ are related to the $4 \mathrm{D}$ central charge $c_{4 \mathrm{D}}$ and the flavor central charge $k_{F}$ as $^{2}$

$$
c_{2 \mathrm{D}}=-12 c_{4 \mathrm{D}}, \quad k_{2 \mathrm{D}}=-k_{F} .
$$

(ii) The (normalized) vacuum character $\chi_{0}(q)$ of $2 \mathrm{D}$ chiral algebra is the 4D Schur index $\mathcal{I}(q),{ }^{3}$

$$
\chi_{0}(q)=\mathcal{I}(q) .
$$

The second relation provides a powerful tool to study the spectrum of Schur operators of 4D theories even when the 4D theory does not admit a Lagrangian description.

We consider two types of AD theories engineered from M5 brane and propose that their chiral algebras take the following form:

(i) The first class $J^{b}[k]$ is engineered using a single irregular singularity, and we consider those theories without any flavor symmetry. The chiral algebra is conjectured to be the following coset:

$$
\mathcal{A}=\frac{J_{l} \bigoplus J_{1}}{J_{l+1}}, \quad l=-\frac{k h-b}{k},
$$

\footnotetext{
${ }^{1} \mathrm{~A}$ very novel twisting procedure is needed to get those 4D/2D mappings [6].

${ }^{2}$ Our normalization of $k_{F}$ is half of that of $[6,8]$.

${ }^{3}$ The definition of Schur and other indices are summarized in the Appendix.
} 
with $h$ being the dual Coxeter number of Lie algebra $J$. This is the minimal model of $\mathrm{W}$ algebra of type $\mathrm{J}$ [19]: $W^{J}\left(p^{\prime}, p\right)=W^{J}(b+k, b)$.

(ii) The second class $\left(J^{b}[k], F\right)$ is engineered using a single irregular singularity (no mass parameters) and a full regular singularity. The chiral algebra is conjectured to be the affine Kac-Moody algebra,

$$
\mathcal{A}=J_{-k_{F}}, \quad k_{F}=h-\frac{b}{b+k} .
$$

\section{AD THEORY WITHOUT FLAVOR SYMMETRY}

Let us start with the theory engineered using one irregular singularity. The irregular singularity has been classified in [18] and the corresponding theory is called $J^{b}[k]$; here $J$ denotes the type of six-dimensional $(2,0)$ theory and $b$ is a number specifying types. The classification is achieved using the classification of irregular solution to Hitchin's equation

$$
\Phi=\frac{T}{z^{2+k / b}}+\cdots .
$$

The corresponding theory can also be realized by putting type IIB string theory on a threefold singularity whose form associated with the $J^{b}[k]$ theory is listed in Table I. We are interested in cases where there is no flavor symmetry. This would put constraints on integer $k$ in each class; see Table II. The threefold singularity has a manifest $C^{*}$ action

$$
f\left(\lambda^{q_{i}} z_{i}\right)=\lambda f\left(z_{i}\right)
$$

TABLE I. Threefold singularities corresponding to our irregular punctures [18], where $\mu_{0}$ is the dimension of charge lattice. When $b=h$ with $\mathrm{h}$ the dual Coxeter number, such theories are also called $\left(J, A_{k-1}\right)$ which were first studied in [2].

\begin{tabular}{lccc}
\hline \hline$J$ & Singularity & $b$ & $\mu_{0}$ \\
\hline$A_{N-1}$ & $x_{1}^{2}+x_{2}^{2}+x_{3}^{N}+z^{k}=0$ & $N$ & $(N-1)(k-1)$ \\
& $x_{1}^{2}+x_{2}^{2}+x_{3}^{N}+x_{3} z^{k}=0$ & $N-1$ & $N(k-1)+1$ \\
$D_{N}$ & $x_{1}^{2}+x_{2}^{N-1}+x_{2} x_{3}^{2}+z^{k}=0$ & $2 N-2$ & $N(k-1)$ \\
& $x_{1}^{2}+x_{2}^{N-1}+x_{2} x_{3}^{2}+z^{k} x_{3}=0$ & $N$ & $2 k(N-1)-N$ \\
$E_{6}$ & $x_{1}^{2}+x_{2}^{3}+x_{3}^{4}+z^{k}=0$ & 12 & $6 k-6$ \\
& $x_{1}^{2}+x_{2}^{3}+x_{3}^{4}+z^{k} x_{3}=0$ & 9 & $8 k-6$ \\
& $x_{1}^{2}+x_{2}^{3}+x_{3}^{4}+z^{k} x_{2}=0$ & 8 & $9 k-6$ \\
$E_{7}$ & $x_{1}^{2}+x_{2}^{3}+x_{2} x_{3}^{3}+z^{k}=0$ & 18 & $7 k-7$ \\
& $x_{1}^{2}+x_{2}^{3}+x_{2} x_{3}^{3}+z^{k} x_{3}=0$ & 14 & $9 k-7$ \\
$E_{8}$ & $x_{1}^{2}+x_{2}^{3}+x_{3}^{5}+z^{k}=0$ & 30 & $8 k-8$ \\
& $x_{1}^{2}+x_{2}^{3}+x_{3}^{5}+z^{k} x_{3}=0$ & 24 & $10 k-8$ \\
& $x_{1}^{2}+x_{2}^{3}+x_{3}^{5}+z^{k} x_{2}=0$ & 20 & $12 k-8$ \\
\hline \hline
\end{tabular}

TABLE II. Constraint on $k$ so that $J^{b}[k]$ has no flavor symmetry.

\begin{tabular}{lccc}
\hline \hline $\mathcal{T}$ & \multicolumn{1}{c}{} \\
\hline$A_{N-1}^{N}[k]$ & $(k, N)=1$ & $A_{N-1}^{N-1}[k]$ & No solution \\
$D_{N}^{2 N-2}[k]$ & $k \neq 2 n$ & $D_{N}^{N}[k]$ & $N=2^{m}(2 i+1), k \neq 2^{m} n$ \\
$E_{6}^{12}[k]$ & $k \neq 3 n$ & $E_{6}^{9}[k]$ & $k \neq 9 n$ \\
$E_{6}^{8}[k]$ & No solution & $E_{7}^{18}[k]$ & $k \neq 2 n$ \\
$E_{7}^{14}[k]$ & $k \neq 2 n, n>1$ & $E_{8}^{30}[k]$ & $k \neq 30 n$ \\
$E_{8}^{24}[k]$ & $k \neq 24 n$ & $E_{8}^{20}[k]$ & $k \neq 20 n$ \\
\hline \hline
\end{tabular}

This $C^{*}$ action is related to the $U(1)_{R}$ symmetry of $\mathcal{N}=2$ SCFT. The Seiberg-Witten curves for these theories can be found from the miniversal deformation of the singularity $[20,21]$.

The 4D central charge can be computed using the following formula [22]:

$$
a=\frac{R(A)}{4}+\frac{R(B)}{6}+\frac{5 r}{24}, \quad c=\frac{R(B)}{3}+\frac{r}{6},
$$

where $r$ is the rank of the Coulomb branch, and for our theories [21],

$$
R(A)=\sum_{\left[u_{i}\right]>1}\left(\left[u_{i}\right]-1\right), \quad R(B)=\frac{1}{4} \mu u_{\max },
$$

in which $\mu$ is the dimension of the charge lattice and $u_{\max }$ is the maximal scaling dimension of Coulomb branch operators, both of which can be expressed in terms of the weights $q_{i}$ 's,

$$
\mu=\prod\left(\frac{1}{q_{i}}-1\right), \quad u_{\max }=\frac{1}{\sum q_{i}-1} .
$$

Other quantities appearing in the central charge formula are also easy to compute,

$$
r=\frac{\mu_{0}}{2}, \quad \mu=\mu_{0} .
$$

We list $\mu_{0}$ in Table I and central charge $c_{4 \mathrm{D}}$ in Table III.

To illustrate how to use the central charge formula, we consider $A_{2 N}^{2 N+1}[2]$ theory with $N$ an integer. Its Coulomb branch operators have conformal dimension $\{(2 N+4)$ / $(2 N+3),(2 N+6) /(2 N+3), \ldots,(4 N+2) /(2 N+3)\}$. Therefore,

$$
R(A)=\frac{N^{2}}{2 N+3}, \quad R(B)=\frac{N(4 N+2)}{2(2 N+3)}
$$


TABLE III. 4D central charges of theory $J^{b}[k]$ without flavor symmetry.

\begin{tabular}{lccc}
\hline \hline $\mathcal{T}$ & $c_{4 \mathrm{D}}$ & $\mathcal{T}$ & $c_{4 \mathrm{D}}$ \\
\hline$A_{N-1}^{N}[k]$ & $\frac{(N-1)(k-1)(N+k+N k)}{12(N+k)}$ & $A_{N-1}^{N-1}[k]$ & $\frac{(N k-N+1)(N+k+N k-1)}{12(N-1+k)}$ \\
$D_{N}^{2 N-2}[k]$ & $\frac{N(k-1)(-2-k+2 N+2 k N)}{12(-2+k+2 N)}$ & $D_{N}^{N}[k]$ & $\frac{((N-1) 2 k-N)(N+k(2 N-1))}{12(k+N)}$ \\
$E_{6}^{12}[k]$ & $\frac{(k-1)(12+13 k)}{2(12+k)}$ & $E_{6}^{9}[k]$ & $\frac{(4 k-3)(13 k+9)}{6(9+k)}$ \\
$E_{6}^{8}[k]$ & $\frac{(3 k-2)(13 k+8)}{4(8+k)}$ & $E_{7}^{18}[k]$ & $\frac{7(k-1)(19 k+18)}{12(18+k)}$ \\
$E_{7}^{14}[k]$ & $\frac{(9 k-7)(19 k+14)}{12(14+k)}$ & $E_{8}^{30}[k]$ & $\frac{2(k-1)(30+31 k)}{3(30+k)}$ \\
$E_{8}^{24}[k]$ & $\frac{(5 k-4)(24+31 k)}{6(24+k)}$ & $E_{8}^{20}[k]$ & $\frac{(3 k-2)(20+31 k)}{3(20+k)}$ \\
\hline \hline
\end{tabular}

and then

$$
c_{4 \mathrm{D}}\left(A_{N-1}^{N}[2]\right)=\frac{1}{6} \frac{N(6 N+5)}{2 N+3} .
$$

\section{A. 2D chiral algebra}

It was realized in [4] that the chiral algebra of $A_{N-1}^{N}[k]$ is $W^{A_{N-1}}(N+k, N)$ minimal model. What is really important to us is that such model can be realized as the coset [23]

$$
\mathcal{A}=\frac{S U(N)_{l} \bigoplus S U(N)_{1}}{S U(N)_{l+1}} .
$$

Here $l=-\frac{(k-1) N}{k}$. Motivated by this coset realization of chiral algebra, we would like to conjecture that the 2D chiral algebra for all our model $J^{b}[k]$ can be realized as the following diagonal coset model:

$$
\mathcal{A}=\frac{\mathfrak{g}_{l} \bigoplus \mathfrak{g}_{1}}{\mathfrak{g}_{l+1}}, \quad \mathfrak{g}=J, \quad l=-\frac{h k-b}{k} .
$$

Here $h$ is the dual Coxeter number. The 2D central charge of our coset model is

$$
c_{2 \mathrm{D}}[J, l, h]=\frac{l \operatorname{dim} J}{l+h}+\frac{\operatorname{dim} J}{1+h}-\frac{(l+1) \operatorname{dim} J}{l+1+h} .
$$

One can check the 2D central charge is related to 4D central charge as in formula 1. The Lie algebra data are shown in Table IV.

We would like to make several comments which are as follows:

(i) For our theory $J^{b}[k]$, the 2D chiral algebra is actually the minimal model of the $\mathrm{W}$ algebra and can be denoted as $W^{J}\left(p^{\prime}, p\right)=W^{J}(b+k, b)$ [19]. The 2D central charge takes the following form:

$$
c_{2 \mathrm{D}}=\operatorname{rank}(J)\left[1-h(h+1) \frac{\left(p^{\prime}-p\right)^{2}}{p p^{\prime}}\right] .
$$

TABLE IV. Lie algebra data.

\begin{tabular}{lccc}
\hline \hline $\mathrm{J}$ & $\operatorname{dim}(J)$ & $\operatorname{rank}(J)$ & $\mathrm{h}$ \\
\hline$A_{N-1}$ & $N^{2}-1$ & $N-1$ & $N$ \\
$D_{N}$ & $2 N^{2}-N$ & $N$ & $2 N-2$ \\
$E_{6}$ & 78 & 6 & 12 \\
$E_{7}$ & 133 & 7 & 18 \\
$E_{8}$ & 248 & 8 & 30 \\
\hline \hline
\end{tabular}

(ii) To match the central charges of 4D and 2D, there are two choices of the levels $l, l_{1}$ which satisfy the condition

$$
l+l_{1}=-(2 h+1) .
$$

These two levels have the property: $l+h>0$ and $l_{1}+h<0$. The choice of $l$ makes the computation easier, and it would be interesting to consider the other choice too.

(iii) For the $A_{N-1}^{N}[k]$ type theory, they can be either engineered using $6 \mathrm{D} A_{N-1}$ theory or $6 \mathrm{D} A_{k-1}$ theory. This implies that the two cosets should be isomorphic by exchanging $k$ and $N$; indeed, such isomorphism has been confirmed in $[24,25]$.

(iv) The $D_{4}^{6}[3]$ theory is also called the $\left(A_{2}, D_{4}\right)$ theory, and this theory is the same as the theory considered in [12]. We have found a different realization of the chiral algebra from the one found in [12]. It would be interesting to compare them.

Consider the chiral algebra $\mathcal{A}\left(J^{h}[k]\right)=W^{J}\left[p^{\prime}, p\right]=$ $W^{J}[h+k, h]$ with $(k, h)=1$. The vacuum character takes the following simple form $[19,26]^{4}$ :

$$
\chi(q)=\frac{1}{\eta(\tau)^{r}} \sum_{\omega \in \hat{W}} \epsilon(w) q^{\frac{1}{2 p p^{\prime}}\left|p^{\prime} w\left(\Lambda^{+}+\rho\right)-p\left(\Lambda^{-}+\rho\right)\right|^{2}}
$$

Here $r$ is the rank of the Lie algebra $J, \hat{W}$ is the affine Weyl group, and $\epsilon(w)$ is the signature of the affine Weyl group element. $\Lambda^{+} \in P_{+}^{0}$ and $\Lambda^{-} \in P_{+}^{k}$ are principle admissible weights such that

$$
\frac{1}{12} r h(h+1)\left(p^{\prime}-p\right)^{2}=\left|p^{\prime}\left(\Lambda^{+}+\rho\right)-p\left(\Lambda^{-}+\rho\right)\right|^{2} .
$$

We have the solution $\Lambda^{+}=0$ and $\Lambda^{-}=(k, 0, \ldots, 0)$. Substituting into the character formula, we get the Schur index of our 4D theory $J^{b}[k]$. Other cases with $b \neq h$ will be considered in a separate publication.

\footnotetext{
${ }^{4}$ For $J=A$, the character is already considered in [4].
} 


\section{ARGYRES-DOUGLAS MATTER}

Argyres-Douglas matters are defined as those isolated $\mathcal{N}=2$ SCFTs with the following properties: (i) the Coulomb branch operators carry fractional scaling dimensions and (ii) they carry non-Abelian flavor symmetries. They can be engineered by putting M5 branes on a sphere with one irregular and one regular puncture. These theories can be labeled as $\left(J^{b}[k], Y\right)$ [18]. The irregular singularity is labeled by $J^{b}[k]$ and the regular singularity is labeled by $Y$ $[27,28] .{ }^{5}$ We focus on the theory whose irregular singularity does not have mass parameters.

Let us consider the simplest case where the regular puncture is full [28], and then our theory can be labeled as $\left(J^{b}[k], F\right)$. The 4D central charge can be computed using formula (7), and we have the following observation:

$$
u_{\max }=\frac{1+(h-1) q(z)}{\sum q_{i}-1}=u_{0 \max }+\frac{(h-1) q(z)}{\sum q_{i}-1} .
$$

Here $q_{i}$ are the weights of the coordinates $x_{i}, z$ of the coordinates appearing in the threefold singularity of the irregular puncture. Other quantities appearing in the central charge formula are also easy to compute,

$r=\frac{\mu_{0}}{2}+\frac{\operatorname{dim}(J)-\operatorname{rank}(J)}{2}, \quad \mu=\mu_{0}+\operatorname{dim}(J)$.

The flavor central charge is conjectured to be equal to the maximal scaling dimension of the Coulomb branch operators,

$$
k_{F}\left(J^{b}[k], F\right)=u_{\max }=h-\frac{b}{b+k},
$$

where $h$ is the dual Coxeter number of Lie algebra $J$. The central charges of 4D theories are listed in Table V.

\section{A. 2D chiral algebra}

We conjecture that the chiral algebra associated with the $\mathrm{AD}$ matter $\left(J^{b}[k], F\right)$ is the affine Kac-Moody algebra,

$$
\mathcal{A}=J_{-k_{F}}
$$

One can easily check the 2D central charge and 4D central charge are related in the correct way as in Eq. (1). The 2D vacuum character and hence the 4D Schur index can be computed using the formula presented in [30] if $b=h$, $(b, k)=1$; other cases would be considered elsewhere.

Now let us study some examples. Consider the simplest model $\left(A_{2 N}^{2 N+1}[-2 N+1], F\right)=D_{2}[S U(2 N+1)]$. The $2 \mathrm{D}$ chiral algebra is the Kac-Moody algebra $S U(2 N+1)_{-2 N+1}$. For $D_{2}[S U(5)]$, the first few orders of vacuum character of

\footnotetext{
${ }^{5}$ When $b=h, J \neq A$, those theories are first studied in [29].
}

TABLE V. The central charge $c_{4 \mathrm{D}}$ and flavor central charge $k_{F}$ for Argyres-Douglas matter.

\begin{tabular}{lcc}
\hline \hline Theory & $c_{4 \mathrm{D}}$ & $k_{F}$ \\
\hline$\left(A_{N-1}^{N}[k], F\right)$ & $\frac{1}{12}(N+k-1)\left(N^{2}-1\right)$ & $\frac{N(N+k-1)}{N+k}$ \\
$\left(A_{N-1}^{N-1}[k], F\right)$ & $\frac{(N+1)\left[N^{2}+N(k-2)+1\right]}{12}$ & $\frac{(N-1)^{2}+k N}{N+k-1}$ \\
$\left(D_{N}^{2 N-2}[k], F\right)$ & $\frac{1}{12} N(2 N-1)(2 N+k-3)$ & $\frac{(2 N-2)(2 N+k-3)}{2 N-2+k}$ \\
$\left(D_{N}^{N}[k], F\right)$ & $\frac{(2 N-1)[2 k(N-1)+N(2 N-3)]}{12}$ & $\frac{2 k(N-1)+N(2 N-3)}{N+k}$ \\
$\left(E_{6}^{12}[k], F\right)$ & $\frac{13(k+11)}{2}$ & $\frac{12(k+11)}{k+12}$ \\
$\left(E_{6}^{9}[k], F\right)$ & $\frac{13}{6}(33+4 k)$ & $12-\frac{9}{k+9}$ \\
$\left(E_{6}^{8}[k], F\right)$ & $\frac{13}{4}(22+3 k)$ & $12-\frac{8}{k+8}$ \\
$\left(E_{7}^{18}[k], F\right)$ & $\frac{133}{12}(17+k)$ & $\frac{18(k+17)}{k+18}$ \\
$\left(E_{7}^{14}[k], F\right)$ & $\frac{19}{12}(119+9 k)$ & $18-\frac{14}{k+14}$ \\
$\left(E_{8}^{30}[k], F\right)$ & $\frac{62}{3}(29+k)$ & $\frac{30(k+29)}{k+30}$ \\
$\left(E_{8}^{24}[k], F\right)$ & $\frac{31}{6}(116+5 k)$ & $30-\frac{24}{k+24}$ \\
$\left(E_{8}^{20}[k], F\right)$ & $\frac{31}{3}(58+3 k)$ & $30-\frac{20}{k+20}$ \\
\hline \hline
\end{tabular}

the affine Kac-Moody algebra are computed using Eq. (18) and we found that the equation can be written in a nice compact form using the plethystic exponential

$$
\mathcal{I}_{D_{2}[S U(5)]}=\operatorname{PE}\left[\left(q+q^{3}+q^{5}+q^{7}+\cdots\right) \chi_{a d j}^{S U(5)}\right],
$$

and for $D_{2}[S U(7)]$, we have

$$
\mathcal{I}_{D_{2}[S U(7)]}=\operatorname{PE}\left[\left(q+q^{3}+\cdots\right) \chi_{a d j}^{S U(7)}\right],
$$

where $\chi_{a d j}^{\mathcal{G}}$ is the character of the adjoint representation of Lie algebra $\mathcal{G}$ and PE means the plethystic exponential,

$$
\mathrm{PE}[x]=\exp \left[\sum_{n=1}^{\infty} \frac{1}{n} x^{n}\right]=\frac{1}{1-x} .
$$

We conjecture that the Schur index of $D_{2}[S U(2 N+1)]$ is

$$
\mathcal{I}_{D_{2}[S U(2 N+1)]}=\operatorname{PE}\left[\frac{q}{1-q^{2}} \chi_{a d j}^{S U(2 N+1)}\right] .
$$

Notice this is the same as the Schur index of half-hypers in adjoint representation of $S U(2 N+1)$ with $q$ substituted by $q^{2}$ [31,32], or in 2D language the vacuum character of $S U(2 N+1)_{-2 N+1}$ is the partition function of free symplectic bosons in adjoint representation of $S U(2 N+1)$ with $q$ replaced by $q^{2}$.

The Schur index of $D_{2}[S U(2 N+1)]$ [Eq. (27)] also reproduces the correct chiral ring relations of the theory. One can check this by comparing with the Hall-Littlewood (HL) index of the same theory. The HL index of the original 4D theory is the same as the Coulomb branch Hilbert series 


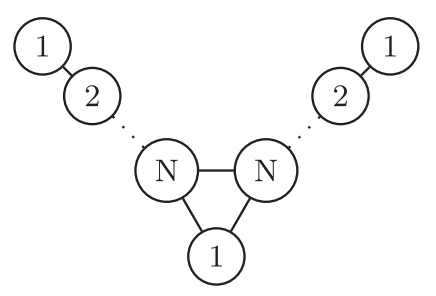

FIG. 1. The 3D mirror quiver of $D_{2}(S U(2 N+1))$ theory.

of the mirror theory [32-34]. If the mirror theory admits a quiver description, one can compute its Coulomb branch Hilbert series following the method in $[33,34]$.

For example, the quiver of the mirror theory of $D_{2}[S U(2 N+1)]$ is shown in Fig. 1 [35]. The Coulomb branch Hilbert series $H_{T(S U(N))}$ of the two quiver tails in the top part of Fig. 1 has been computed explicitly (i.e., Eq. (3.9) of [34]); one can then glue two copies of $H_{T(S U(N))}$ with the rest part of the quiver to get the full $\mathrm{HL}$ index of $D_{2}[S U(2 N+1)]$. For example, the HL index of $D_{2}[S U(5)]$ is

$$
\mathrm{HL}_{D_{2}[S U(5)]}=\mathrm{PE}\left[\chi_{\mathbf{2 4}} t-\left(1+\chi_{\mathbf{2 4}}\right) t^{2}+\chi_{\mathbf{2 4}} t^{3}+\ldots\right],
$$

which means in the chiral ring of $D_{2}[S U(5)]$ there are dimension 2 and 6 generators in $\mathbf{2 4}$ (adjoint) representation of $S U(5)$ together with dimension 4 relations in $\mathbf{2 4}+\mathbf{1}$ representation and more. Exactly same generators and relations can also be extracted from the Schur index (24) by noticing that the Schur indices of dimension $d$ chiral operator (relations) are $\frac{q^{d / 2}}{1-q}\left(-\frac{q^{d / 2}}{1-q}\right)$ instead of $t^{d / 2}\left(-t^{d / 2}\right)$ in HL index and stress tensor multiplet contributes $\frac{q^{2}}{1-q}$ to the Schur index but not present in HL index. One can also check that the Schur and HL indices of $D_{2}[S U(7)]$ produce the same generators and relations of the chiral ring at lower dimension.

The Schur index can also be computed from the trace of the monodromy built from the Bogomolnyi-PrasadSommerfield (BPS) spectrum $[2,4,5]$. For $D_{2}[S U(2 N+1)]$ theory, the minimal chamber has $2 N(2 N+1)$ BPS states using the tools developed in [36,37]. Unfortunately, for $D_{2}[S U(5)]$ theory, the minimal chamber has 20 states; we are not able to compute the trace of the monodromy due to limiting computing power.

\section{DISCUSSIONS}

The chiral algebra for theory considered in this paper is strikingly simple, and it is closed related to the sixdimensional construction; for example, the Lie algebra appearing in the coset model and affine Kac-Moody algebra are precisely the type of $6 \mathrm{D}(2,0)$ theory we start with. It would be interesting to interpret why this same Lie algebra appears in 2D chiral algebra description.
In this paper, we have considered only those irregular singularities without mass parameters. For other type of irregular singularities, one usually has a set of extra $U(1)^{f}$ flavor symmetries, and the 2D chiral algebra should have extra generators corresponding to the moment map and other Higgs branch generators of $U(1)^{f}$ symmetries. The 4D central charge formula takes the following form:

$$
12 c_{4 \mathrm{D}}=-c_{2 \mathrm{D}}-f .
$$

Here $c_{2 \mathrm{D}}$ is the central charge from the coset or the affine Kac-Moody part. The above observation motivates the following conjecture about the chiral algebra of the general case: one has the same coset or affine Kac-Moody factor, and we add extra generator corresponding to Higgs branch operators of $U(1)^{f}$ factor, and the 2D central charge of this piece is $-f$. For example, $D_{2}[S U(2 N)]$ is $S U(N)$ SQCD with $N_{f}=2 N$, according to our proposal, the chiral algebra consists of a affine Kac-Moody factor $S U(2 N)_{-N}$, and we should have extra generators corresponding to the extra $U(1)$ flavor symmetries. This structure agrees with the conjecture made in [6].

We can gauge $\mathrm{AD}$ matter to form new $\mathcal{N}=2$ SCFT. Since we know the chiral algebra of the AD matter and the gluing procedure of the chiral algebra, it would be interesting to find the chiral algebra for theories built from gauging $\mathrm{AD}$ matter. It would be also interesting to find out the chiral algebra of the general $\mathcal{N}=2$ SCFT engineered from threefold singularity [21].

The $J^{b}[k]$ theory can be derived by closing off the full puncture of $\left(J^{b}[k], F\right)$, and it is argued in [6] that the closing off puncture procedure corresponds to Drinfeld-Sokolov (DS) reduction for corresponding $2 \mathrm{D}$ chiral algebra. The chiral algebra of $J^{b}[k]$ theory is W algebra $W^{J}(b+k, k)$, and indeed it can be derived from DS reduction from the affine Kac-Moody algebra $J_{-h+b /(b+k)}$ [26] which is precisely the chiral algebra of the theory $\left(J^{b}[k], F\right) .^{6}$

\section{ACKNOWLEDGMENTS}

The work of S.-T. Y. was supported by NSF Grants No. DMS-1159412, No. PHY-0937443, and No. DMS0804454. The work of D. X. and W. Y. was supported by Center for Mathematical Sciences and Applications at Harvard University. We would like to thank B. Lian, S. Cecotti, L. Rastelli, J. Song,Y. Wang, and especially C.Vafa for invaluable discussions.

\section{APPENDIX: SOME FACTS ABOUT $\mathcal{N}=2$ INDEX}

The generic representation of a $4 \mathrm{D} \mathcal{N}=2$ SCFT is labeled by the states $\left|E, R, r, j_{1}, j_{2}\right\rangle$. Here $E$ is the scaling dimension, $R$ is the $S U(2)_{R}$ quantum number, $r$ is the $U(1)_{R}$ quantum number, and $j_{1}, j_{2}$ are left and right spins.

\footnotetext{
${ }^{6}$ We thank L. Rastelli for this very interesting observation.
} 
The superconformal index for $\mathcal{N}=2$ SCFTs is a refined Witten index on $S^{3} \times S^{1}$,

$$
\mathcal{I}=\operatorname{Tr}(-1)^{F} p^{\frac{1}{2}\left(E+2 j_{1}-2 R-r\right)} q^{\frac{1}{2}\left(E-2 j_{1}-2 R-r\right)} t^{R+r},
$$

where the trace is over BPS states satisfying the condition

$$
E-2 j_{2}-2 R+r=0,
$$

which is annihilated by $\tilde{Q}_{1-}$ supercharge. Different limits of the superconformal index are sensitive to different superconformal multiplets. In this work, we are interested in the following two limits.

The first is the Schur limit $q=t$ with $p$ arbitrary. The result is independent of fugacity $p$ automatically. In a trace formula, the Schur index can be written as

$$
\mathcal{I}_{\text {Schur }}=\operatorname{Tr}_{\text {Schur }}(-1)^{F} q^{E-R},
$$

which traces over BPS states satisfying an additional condition

$$
E+2 j_{1}-2 R-r=0,
$$

besides the original condition which is used to define the index. They are annihilated by $Q_{1+}$ and $\tilde{Q}_{1 \text { - supercharge. }}$ The Schur index counts Higgs branch operators, stress tensor, and other multiplets and is mapped to the vacuum character of the corresponding VOA.

The second is the Hall-Littlewood limit $q, p \rightarrow 0$ with $t$ fixed. The trace formula is

$$
\mathcal{I}_{\mathrm{HL}}=\operatorname{Tr}_{\mathrm{HL}}(-1)^{F} \tau^{2(E-R)} .
$$

The trace is restricted to states with

$$
j_{1}=0, \quad j_{2}=r, \quad E=2 R+r
$$

and is annihilated by supercharges $Q_{1+}, Q_{1-}$, and $\tilde{Q}_{1 \dot{-}}$.
[1] L. F. Alday, D. Gaiotto, and Y. Tachikawa, Lett. Math. Phys. 91, 167 (2010).

[2] S. Cecotti, A. Neitzke, and C. Vafa, arXiv:1006.3435.

[3] A. Iqbal and C. Vafa, Phys. Rev. D 90, 105031 (2014).

[4] C. Cordova and S.-H. Shao, J. High Energy Phys. 01 (2016) 040.

[5] S. Cecotti, J. Song, C. Vafa, and W. Yan, J. High Energy Phys. 11 (2017) 013.

[6] C. Beem, M. Lemos, P. Liendo, W. Peelaers, L. Rastelli, and B. C. van Rees, Commun. Math. Phys. 336, 1359 (2015).

[7] D. Gaiotto, G. W. Moore, and Y. Tachikawa, Prog. Theor. Exp. Phys. 2013, 013B03 (2013).

[8] C. Beem, W. Peelaers, L. Rastelli, and B. C. van Rees, J. High Energy Phys. 05 (2015) 020.

[9] M. Lemos and W. Peelaers, J. High Energy Phys. 02 (2015) 113.

[10] M. Buican and T. Nishinaka, J. Phys. A 49, 015401 (2016).

[11] J. Song, J. High Energy Phys. 02 (2016) 045.

[12] M. Buican and T. Nishinaka, J. Phys. A 49, 465401 (2016).

[13] T. Nishinaka and Y. Tachikawa, J. High Energy Phys. 09 (2016) 116.

[14] P. C. Argyres and M. R. Douglas, Nucl. Phys. B448, 93 (1995).

[15] D. Gaiotto, G. W. Moore, and A. Neitzke, arXiv:0907.3987.

[16] G. Bonelli, K. Maruyoshi, and A. Tanzini, J. High Energy Phys. 02 (2012) 031.

[17] D. Xie, J. High Energy Phys. 01 (2013) 100.

[18] Y. Wang and D. Xie, Phys. Rev. D 94, 065012 (2016).

[19] P. Bouwknegt and K. Schoutens, Phys. Rep. 223, 183 (1993).
[20] A. D. Shapere and C. Vafa, arXiv:hep-th/9910182.

[21] D. Xie and S.-T. Yau, arXiv:1510.01324.

[22] A. D. Shapere and Y. Tachikawa, J. High Energy Phys. 09 (2008) 109.

[23] F. A. Bais, P. Bouwknegt, M. Surridge, and K. Schoutens, Nucl. Phys. B304, 371 (1988).

[24] A. Kuniba, T. Nakanishi, and J. Suzuki, Nucl. Phys. B356, 750 (1991).

[25] D. Altschuler, M. Bauer, and H. Saleur, J. Phys. A 23, L789 (1990).

[26] E. Frenkel, V. Kac, and M. Wakimoto, Commun. Math. Phys. 147, 295 (1992).

[27] D. Gaiotto, J. High Energy Phys. 08 (2012) 034.

[28] O. Chacaltana, J. Distler, and Y. Tachikawa, Int. J. Mod. Phys. A 28, 1340006 (2013).

[29] S. Cecotti and M. Del Zotto, J. High Energy Phys. 01 (2013) 191.

[30] V. G. Kac and M. Wakimoto, Proc. Natl. Acad. Sci. U.S.A. 85, 4956 (1988).

[31] A. Gadde, L. Rastelli, S. S. Razamat, and W. Yan, Phys. Rev. Lett. 106, 241602 (2011).

[32] A. Gadde, L. Rastelli, S. S. Razamat, and W. Yan, Commun. Math. Phys. 319, 147 (2013).

[33] S. Cremonesi, A. Hanany, and A. Zaffaroni, J. High Energy Phys. 01 (2014) 005.

[34] S. Cremonesi, A. Hanany, N. Mekareeya, and A. Zaffaroni, J. High Energy Phys. 09 (2014) 178.

[35] D. Xie and S.-T. Yau, arXiv:1602.03529.

[36] D. Xie, arXiv:1203.4573.

[37] D. Xie, Adv. Theor. Math. Phys. 20, 405 (2016). 